\title{
COVID-19 in a dialysis center in Milan from March to June 2020: understanding how to respond to the second wave of the pandemic
}

\author{
Andrea Galassi ${ }^{1,2} \cdot$ Lorenza Magagnoli $^{1,2} \cdot$ Mario Cozzolino $^{1,2} \cdot$ on behalf of the COVID-19 Renal Working Group at \\ ASST Santi Paolo \& Carlo in Milan
}

Published online: 3 February 2021

(c) Italian Society of Nephrology 2021

When the pandemic struck, it was clear that maintenance haemodialysis (MHD) patients were at high risk of being infected with the acute respiratory distress syndrome associated with the coronavirus (SARS-CoV-2) [1]. Nephrologists were responsible for screening procedures for counteracting a SARS-CoV-2 outbreak in haemodialysis facilities, and for the management of cases in ambulatory and in-hospital settings. Although the mortality risk for maintenance haemodialysis patients was higher than for the general population $[1,2]$, clinical phenotypes of COVID-19 were heterogeneous in this cohort, ranging from asymptomatic to fatal [2-5]. Advanced age, cardiovascular disease (CVD), multimorbidities and frailty, thought to increase vulnerability to COVID-19 complications, were inconsistently associated with mortality in dialysis patients [2-5], making the trend of the disease in individual cases unpredictable. These factors, and the lack of an effective treatment, meant that nephrologists needed to find equitable, time-effective ways of allocating healthcare resources.

As we now face a second pandemic wave, it is imperative that we re-evaluate our recent experience and improve procedures for screening, managing and treating dialysis patients affected by COVID-19.

By discussing the approaches developed by the COVID19 Renal Working Group at the Hospital Santi Paolo \& Carlo (Milan) during the early months of 2020, we tried to answer four crucial questions, which are now being proposed for further discussion.

The members of the COVID-19 Renal Working Group at ASST Santi Paolo and Carlo are listed in the acknowledgements.

Mario Cozzolino

mario.cozzolino@unimi.it

1 Renal and Dialysis Unit, San Paolo Hospital, ASST Santi Paolo e Carlo, Milan, Italy

2 Department of Health Sciences, Renal Division, ASST Santi Paolo E Carlo, University of Milan, Milan, Italy
1. How should HD patients be screened: universal or restricted criteria?

The Santi Paolo \& Carlo hospital group is comprised of the San Paolo (SPH) and San Carlo (SCH) hospitals, each with its own dialysis unit. Maintenance haemodialysis patients at these hospitals were monitored between March 1st and June 30th, 2020. The precautions against viral transmission within the hospitals' haemodialysis facilities have been described elsewhere in detail [6]. Before admittance to the dialysis rooms, the patients' vital parameters (body temperature and peripheral arterial oxygen saturation) were assessed and patients were asked about COVID-19-related symptoms (cough, dyspnoea, dysgeusia, gastrointestinal discomfort), and about contact with a SARS-CoV-2 positive subject. While the purpose of universal screening was to limit viral transmission [7], in accordance with other centres, more restrictive screening criteria were adopted at our institution [2,3]. Patients were categorized in three groups: symptomatic, contact and screening. Those found to be positive based on the check-list described above or that had a fever (body temperature $>37.5^{\circ} \mathrm{C}$ ), hypoxia ( $\mathrm{SpO} 2<92 \%$ in ambient air) or other symptoms were categorized as symptomatic. Maintenance haemodialysis patients testing positive after admission to the emergency room were included in this group. Patients who had received haemodialysis in the same room as a patient who subsequently tested positive, or shared transport with someone in this category, were categorized as contacts. Those admitted to hospital for elective diagnostic or therapeutic procedures not related to COVID-19, were classified as screening patients. A nasopharyngeal swab for real time PCR was routinely performed for symptomatic and screening patients at both hospitals. Contacts systematically received a nasopharyngeal swab only at the San Paolo Hopital. 
Positive outpatients underwent home isolation and received haemodialysis on dedicated shifts for COVID19 patients, as previously described [6]. Positive patients requiring hospitalization were admitted to specific COVID-19 wards at the San Paolo Hospital (COVIDNephrology, COVID-Pneumology) or at the San Carlo Hospital (COVID-Internal Medicine, COVID-Pneumology). Patients received bedside-haemodialysis using portable osmosis in the hospitalization rooms at the San Paolo Hospital and in a dedicated haemodialysis ward at the San Carlo Hospital. Given its potential anti-inflammatory properties, the Theranova ${ }^{\circledR}$ dialyzer (Baxter, USA) was preferred for expanded haemodialysis in positive patients [8].

Of 307 patients, 107 (35\%) were tested. Twenty-one (20\%) of them tested positive, i.e. we found a $7.0 \%$ prevalence of SARS-CoV-2 infection for the entire cohort, in agreement with the $7.6 \%$ rate for maintenance haemodialysis patients in Lombardy (Italy) [1]. The number of positive patients in the symptomatic, contact and screening subgroups were $13(31 \%), 8(19 \%)$ and $0(-)$, respectively (S. Table 1a). The different screening approaches at the two hospitals led to a higher percentage of total patients tested ( $61 \%$ vs $20 \%$ ), patients testing positive (21\% vs $17 \%)$ and contact patients tested $(61 \%$ vs $5 \%)$ at the San Paolo Hospital compared with the San Carlo Hospital. Although screening at the former location detected a $19 \%$ positivity rate among tested contacts, there was no outbreak of the virus at the latter location, where most of the tested patients were symptomatic.
None of the contacts who tested positive were subsequently hospitalized.

On the basis of our experience, therefore, we may suggest that, when healthcare operators have been trained and the facility workflow allows it, screening symptomatic patients alone can be cost-effective [7].

2. Do all MHD patients who test positive for SARS-CoV-2 require hospitalization, or present with respiratory insufficiency and risk death?

Data concerning symptoms, hospitalization, ventilation, and survival were analysed for 28 patients, including 7 cases referred from external facilities (S. Table 1b).

Most patients (79\%) were symptomatic; the most common symptoms were fever, dyspnoea, and cough (S. Table 2). Fourteen patients (50\%) were hospitalized (S. Table $1 \mathrm{~b})$. None of the contacts testing positive needed hospital admission. Five hospitalized patients (36\%) required oxygen via a mask or nasal prongs; 7 (50\%) required non-invasive ventilation (continuous-positiveairway pressure or non-invasive mechanical ventilation, delivered by helmet or full-face mask, respectively) (S. Table 3). None were intubated. The overall mortality rate was $18 \%$ (S. Tables 1,2). Crude mortality was in agreement with other centres [2,3] and was however lower than the $32 \%$ mortality rate for HD patients in Lombardy [1]. Mortality was absent in outpatients, 36\% in hospitalized patients and 57\% in ventilated patients (Fig. 1).
Fig. 1 Mortality rate according to hospitalization and ventilation need

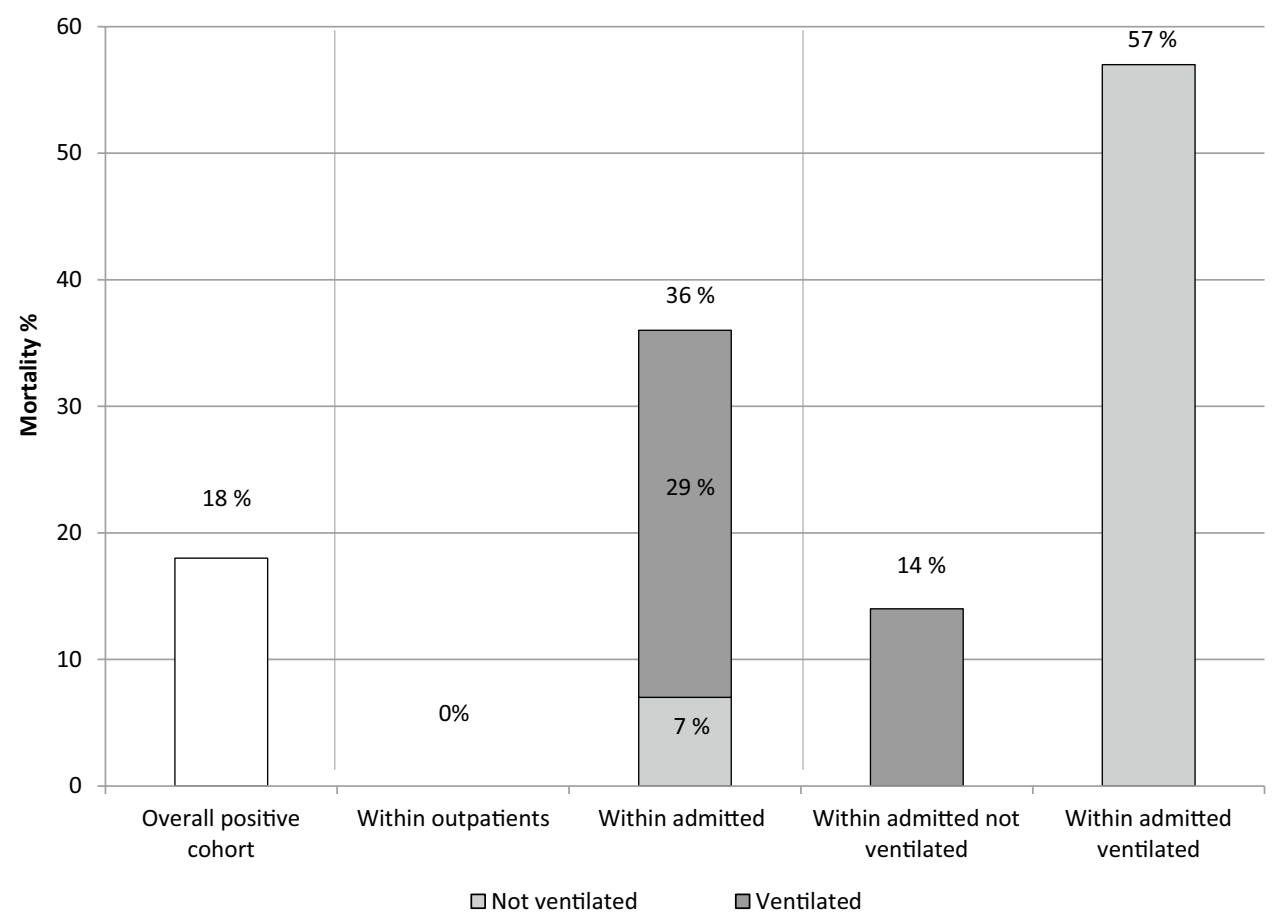


On the basis of our experience we can therefore support the literature data and confirm that COVID-19 presentation in dialysis patients varies considerably [7, 9]. Mortality is almost null in outpatients, higher in hospitalized patients, and even higher in those requiring ventilation. However, maintenance haemodialysis should not represent an independent contraindication for subintensive care in COVID-19 MHD patients.

3. Should outcomes be predicted and resources allocated using an age- and comorbidity-driven approach, clinical presentation or both?

Age and cardiovascular diseases were descriptively associated with hospitalization, ventilation and mortality (S. Table 2, S. Fig. 1). The Charlson Comorbidity Index (CCI) increased between hospitalized, ventilated and deceased patients (S. Table 2), reaching borderline significance as a predictor of outcomes only for univariate models (S. Tables 4, 5). In terms of signs and symptoms, univariate analysis revealed a direct association between hospitalization and fever, dyspnoea and other symptoms. Ventilation and mortality were associated with dyspnoea and lower $\mathrm{SpO} 2$ at presentation. On the basis of multivariate regression, $\mathrm{SpO} 2<97 \%$ predicted ventilation independently from hospitalization; ventilation predicted mortality independently from age and CVD (S. Table 4). Among inflammatory markers, C-reactive protein was associated with hospitalization, ventilation and survival only at univariate analysis (S. Tables 2, 4). A lower eosinophil count was associated with hospitalization (S. Tables 2, 4); eosinopenia improved in patients who were admitted and survived but not in patients who died after being hospitalized (S. Table 6, S. Fig. 2).

On the basis of our experience, and of the review of the literature, we therefore suggest that disease presentation and clinical trend appear to be more reliable predictors of outcome than age and comorbidities [4]. Dyspnoea and even a mild reduction in $\mathrm{SpO} 2$ levels at diagnosis can be taken as strong predictors of respiratory failure and fatal outcomes. Although low comorbidity $(\mathrm{CCI}<7)$ is associated with better survival, clinical presentation should always be taken into account when estimating the prognosis of elderly and high comorbidity patients. Thus, in delivering both sub-intensive and intensive care, younger and low comorbidity dialysis patients with severe COVID-19 should be given priority. Eosinopenia may represent an early sign of infection; its subsequent improvement may conversely be a marker of recovery from COVID-19.

4. How should viral clearance be managed?

Extended viremia has been reported in MHD patients, raising uncertainties about the risk of viral transmission by patients with a persisting positive nasopharyngeal swab, in clinical remission [9]. Longer hospitalization was recently reported in maintenance haemodialysis patients requiring non-invasive ventilation [4]. A prolonged median time for viral clearance of 32 days (IQR 22-46) and for hospital discharge of 49 days (IQR 46-55) was confirmed in our population (S. Fig. 3). A history of hypertension was the only characteristic significantly associated with a longer viral clearance time at sensitivity analysis (S. Fig. 3). Clinical severity and/or impossibility to guarantee home quarantine before viral clearance may have accounted for long in-hospital stays.

On the basis of our experience, and in keeping with the literature, prolonged viral clearance can be expected in MHD patients. Careful evaluation of the patient's social setting is crucial in planning post-hospital quarantine to avoid the saturation of in-hospital healthcare resources.

\section{Conclusions}

The healthcare system was unprepared for the first pandemic wave.

The haemodialysis techniques and treatments which can improve the survival of chronic haemodialysis patients with COVID-19 have not yet been fully assessed; in our experience daily haemodialysis that was delivered at bedside showed a potential life-saving effect.

In facing the second wave, being on chronic dialysis per se should not limit critically ill COVID-19 patients' access to intensive care. However, advanced directives in favour of or against intubation could be pro-actively proposed to frail and elderly dialysis patients or their relatives, respecting the patients' wishes and dignity and contributing to the wise allocation of healthcare resources [5].

Electronic Supplementary Information The online version contains supplementary material available at https://doi.org/10.1007/s4062 0-020-00933-8.

Acknowledgements All the following members of the COVID-19 Renal Working Group at ASST Santi Paolo and Carlo in Milan contributed equally to this work. Renal \& Dialysis Unit San Paolo Hospital: Eliana Fasulo (MD), Ulisse Zoni (MD), Andrea Stucchi (MD), Paola Monciino (MD), Michela Frittoli (MD), Michela Ippolito (Coordinator of Dialysis Nursing Staff). Renal and Dialysis Unit San Carlo Hospital: Chiara Leotta (MD), Antonino Giordano (MD), Roberta Casazza (MD), Matthias Cassia (MD), Giovanni Battista di Tria (Coordinator of Dialysis Nursing Staff).

Honoured acknowledgements are dedicated to the whole renal and dialysis nursing staff, who spent hours close to COVID-19 dialysis patients delivering life-saving therapies on the front lines (, at the best of our chances). 


\section{Compliance with ethical standards}

Conflict of interest The authors declare that they have no conflict of interest.

Ethical approval All procedures performed in studies involving human participants were in accordance with the ethical standards of the institutional and/or national research committee and with the 1964 Helsinki declaration and its later amendments or comparable ethical standards.

Informed consent For this type of study formal consent is not required.

\section{References}

1. Quintaliani G, Reboldi G, Di Napoli A, Nordio M, Limido A, Aucella F (2020) MessaP, Brunori G; Italian Society of Nephrology COVID-19 Research Group. Exposure to novel coronavirus in patients on renal replacement therapy during the exponential phase of COVID-19 pandemic: survey of the Italian Society of Nephrology. J Nephrol. 33(4):725-736

2. Alberici F, Delbarba E, Manenti C et al (2020) A report from the Brescia renal COVID task force on the clinical characteristics and short-term outcome of hemodialysis patients with SARS-CoV-2 infection. Kidney Int 98:20-26
3. Corbett RW, Blakey S, Nitsch D et al (2020) Epidemiology of COVID-19 in an Urban Dialysis Center. J Am Soc Nephrol. https ://doi.org/10.1681/ASN.2020040534

4. Tortonese S, Scriabine I, Anjou L et al (2020) COVID-19 in patients in maintenance dialysis in Paris Region. Kidney Int Rep. https://doi.org/10.1016/j.ekir.2020.07.016

5. Valeri AM, Robbins-Juarez SY, Stevens JS et al (2020) Presentation and outcomes of patients with ESKD and COVID-19. J Am Soc Nephrol 31:1409-1415

6. Ippolito M, Di Tria GB, Aldrigo C et al (2020) Standard procedures in dialysis during the Covid-19 epidemic. G Ital Nefrol 37

7. Kliger AS, Cozzolino M, Vivekanand J et al (2020) (2020) Managing the COVID-19 pandemic: international comparisons in dialysis patients. Kidney Int 98:12-16

8. Cozzolino M, Magagnoli L, Ciceri P et al (2019) Effects of medium cut-off (Theranova) dialyzer on hemodialysis patients: a prospective cross-over study. Clin Kidney J. https://doi. org/10.1093/ckj/sfz155

9. Dudreuilh C, Kumar N, Moxham V et al (2020) De-isolation of COVID-19 positive hemodialysis patients in the outpatient setting: a single-center experience. Kidney Int 98:236-237

Publisher's Note Springer Nature remains neutral with regard to jurisdictional claims in published maps and institutional affiliations. 